\title{
On Modified Mellin Transform of Generalized Functions
}

\author{
S. K. Q. Al-Omari ${ }^{1}$ and Adem Kılıçman ${ }^{2}$ \\ ${ }^{1}$ Department of Applied Sciences, Faculty of Engineering Technology, Al-Balqa Applied University, Amman 11134, Jordan \\ ${ }^{2}$ Department of Mathematics and Institute of Mathematical Research, Universiti Putra Malaysia (UPM), 43400 Serdang, \\ Selangor, Malaysia
}

Correspondence should be addressed to Adem Kılıçman; kilicman@yahoo.com

Received 10 September 2013; Accepted 14 November 2013

Academic Editor: S. A. Mohiuddine

Copyright (C) 2013 S. K. Q. Al-Omari and A. Kılıçman. This is an open access article distributed under the Creative Commons Attribution License, which permits unrestricted use, distribution, and reproduction in any medium, provided the original work is properly cited.

\begin{abstract}
We investigate the modified Mellin transform on certain function space of generalized functions. We first obtain the convolution theorem for the classical and distributional modified Mellin transform. Then we describe the domain and range spaces where the extended modified transform is well defined. Consistency, convolution, analyticity, continuity, and sufficient theorems for the proposed transform have been established. An inversion formula is also obtained and many properties are given.
\end{abstract}

\section{Introduction}

The Mellin transform $\mu$ of a suitably restricted function over $\mathbb{R}_{+}((0, \infty))$ was defined on some strip in the complex plane [1], where many of the transform properties are obtained by applying change of variables to various properties of the Laplace transformation. The Mellin transform is extended to distributions in [1] and to Boehmians in [2].

By combining Fourier and Mellin transforms, the obtained transform is called Fourier-Mellin transform which has many applications in digital signals, image processing, and ship target recognition by sonar system and radar signals as well.

The modified Mellin transform of a suitably restricted function $f$ over $\mathbb{R}_{+}$was introduced by [3]

$$
\mu_{f}^{m}(y)=: \mu^{m} f(y)=: \int_{\mathbb{R}_{+}} f(x) y x^{y-1} d x
$$

as a scale-invariant transform. Then, as earlier, combining the modified Mellin transform with the Fourier transform gives the Fourier-modified Mellin transform [3, Equation 16].

The Mellin-type convolution product of two functions $f$ and $g$ is given by

$$
(f \vee g)(x)=\int_{\mathbb{R}_{+}} f(\tau) g\left(\frac{x}{\tau}\right) \tau^{-1} d \tau .
$$

From [1] it has been noted that

$$
\mu(f \vee g)(y)=\mu_{f}(y) \mu_{g}(y) .
$$

Utilizing the Mellin-type convolution product the following theorem is essential for our next investigations.

Theorem 1 (convolution theorem). Let $\mathscr{L}^{1}$ be the Lebesgue space of integrable functions and $f, g \in \mathscr{L}^{1}$; then

$$
\mu^{m}(f \vee g)(y)=\mu_{f}^{m}(y) \mu_{g}(y),
$$

where $\mu_{f}^{m}$ and $\mu_{g}$ are the Mellin-type and Mellin transforms of $f$ and $g$, respectively.

Proof. By the definition of the Mellin and modified Mellin transforms we have

$$
\begin{aligned}
\mu^{m}(f \vee g)(y) & =\int_{\mathbb{R}_{+}}(f \vee g)(x) y x^{y-1} d x \\
& =\int_{\mathbb{R}_{+}}\left(\int_{\mathbb{R}_{+}} f(\tau) g\left(\frac{x}{\tau}\right) \tau^{-1} d \tau\right) y x^{y-1} d x \\
& =\int_{\mathbb{R}_{+}} y f(\tau) \tau^{-1}\left(\int_{\mathbb{R}_{+}} g\left(\frac{x}{\tau}\right) x^{y-1} d x\right) d \tau .
\end{aligned}
$$


Employing Fubinis theorem, then the substitution $z=x / \tau$ together with simple computations establishes that

$$
\mu^{m}(f \vee g)(y)=\mu_{f}^{m}(y) \mu_{g}(y) .
$$

Hence the theorem is proved.

\section{Modified Mellin Transform of Distribution}

Let $\mu_{a, b}$ be the space of smooth functions $\varphi$ over $\mathbb{R}_{+}$such that [1]

$$
\gamma_{k}(\varphi)=\sup _{x \in \mathbb{R}_{+}}\left|\xi_{a, b}(x) x^{k-1} \frac{d^{k}}{d x^{k}} \varphi(x)\right|
$$

is finite, $k=0,1,2, \ldots$, where

$$
\xi_{a, b}(x) \triangleq \begin{cases}x^{-a}, & \text { for } 0<x \leq 1 \\ x^{-b}, & \text { for } 1<x<\infty\end{cases}
$$

and $a, b \in \mathbb{R}_{+}$.

Then $\mu_{a, b}$ is linear space under addition and multiplication by complex numbers. $\mu_{a, b}$ can also be generated by the multinorms $\left(\gamma_{k}\right)_{0}^{\infty}$ which turns to be a countably multinormed space.

Denote by $\dot{\mu}_{a, b}$ the complete strong dual space of $\mu_{a, b}$; then it is assigned the weak topology. Let $\xi\left(\mathbb{R}_{+}\right)$be the space of smooth functions over $\mathbb{R}_{+}$; then for any $a, b \in \mathbb{R}, \mu_{a, b}$ is dense in $\xi\left(\mathbb{R}_{+}\right)$and the topology assigned to $\mu_{a, b}$ is stronger than that induced on $\mu_{a, b}$ by $\xi\left(\mathbb{R}_{+}\right)$and is identified with a subspace of $\dot{\mu}_{a, b}$.

The straightforward conclusion is that the kernel function $\left(y x^{y-1}\right)$ of $\mu_{f}^{m}$ is a member of $\mu_{a, b}$ for $a \leq \operatorname{Re} y \leq b$.

This usually leads to the following definition: let $f \in \dot{\mu}_{a, b}$; then the distributional transform $\widehat{\mu_{f}^{m}}$ of $f$ is defined as

$$
\widehat{\mu_{f}^{m}}(y) \triangleq\left\langle f(x), y x^{y-1}\right\rangle, \quad y \in \Omega_{f}
$$

where $f \in \dot{\mu}_{a, b}$ and $\Omega_{f}=\{y \in \mathbb{C}: a<\operatorname{Re} y<b\}$.

Theorem 2 (analyticity theorem). Let $f \in \dot{\mu}_{a, b}$; then $\widehat{\mu_{f}^{m}}$ is analytic and

$$
\frac{d^{k}}{d y^{k}} \widehat{\mu_{f}^{m}}(y)=\left\langle f(x), y x^{y-1} \ln ^{k} x+k x^{y-1} \ln x^{k-1}\right\rangle,
$$

where $k$ is nonnegative integer and $y \in \Omega_{f}$.

It is easy for reader to see that $\widehat{\mu_{f}^{m}}$ is injective and linear from $\dot{\mu}_{a, b}$ into $\dot{\mu}_{a, b}$.

The Mellin-type convolution product of $f, g \in \dot{\mu}_{a, b}$ is given as

$$
\langle f \vee g, \varphi\rangle \triangleq\langle f(x),\langle g(t), \varphi(t x)\rangle\rangle
$$

where $\varphi \in \mu_{a, b}$.

From (11) it is clear that $f \vee g$ is a member of $\mu_{a, b}$, for $a \leq b$.
Therefore, denote by $\vartheta\left(\mathbb{R}_{+}\right)$the space of test functions of bounded support over $\mathbb{R}_{+}$; then the convolution product of $f \in \dot{\mu}_{a, b}$ and $g \in \vartheta\left(\mathbb{R}_{+}\right)$can be given as

$$
(f \vee g)(x)=\left\langle f(t), \frac{1}{t} g\left(\frac{x}{t}\right)\right\rangle,
$$

where $x \in \mathbb{R}_{+}$.

\section{Boehmians}

Let $\mathscr{G}$ a group and $\mathcal{S}$ a subgroup of $\mathscr{G}$. We assume that to each pair of elements $f \in \mathscr{G}$ and $\omega \in \mathcal{S}$ is assigned the product $f * g$ such that

(1) if $\omega, \psi \in \mathcal{S}$, then $\omega * \psi \in \mathcal{S}$ and $\omega * \psi=\psi * \omega$;

(2) if $f \in \mathscr{G}$ and $\omega, \psi \in \mathcal{S}$, then $(f * \omega) * \psi=f *(\omega \star \psi)$;

(3) if $f, g \in \mathscr{G}, \omega \in \mathcal{S}$, and $\lambda \in \mathbb{R}$, then

$$
(f+g) * \omega=f * \omega+g * \omega, \quad \lambda(f * \omega)=(\lambda f) * \omega .
$$

Let $\Delta$ be a family of sequences from $\delta$ such that

(1) if $f, g \in \mathscr{G},\left(\delta_{n}\right) \in \Delta$, and $f * \delta_{n}=g * \delta_{n}(n=1,2, \ldots)$, then $f=g$, for all $n$;

(2) if $\left(\omega_{n}\right),\left(\delta_{n}\right) \in \Delta$, then $\left(\omega_{n} * \psi_{n}\right) \in \Delta$.

Elements of $\Delta$ will be called delta sequences.

Consider the class $\mathscr{A}$ of pair of sequences defined by

$$
\mathscr{A}=\left\{\left(\left(f_{n}\right),\left(\omega_{n}\right)\right):\left(f_{n}\right) \subseteq \mathscr{G}^{\mathbb{N}},\left(\omega_{n}\right) \in \Delta\right\},
$$

for each $n \in \mathbb{N}$.

An element $\left(\left(f_{n}\right),\left(\omega_{n}\right)\right) \in \mathscr{A}$ is called a quotient of sequences, denoted by $\left[f_{v} / \omega_{n}\right]$, if $f_{n} * \omega_{m}=f_{m} * \omega_{n}$, for all $n, m \in \mathbb{N}$.

Two quotients of sequences $f_{n} / \omega_{n}$ and $g_{n} / \psi_{n}$ are said to be equivalent, $f_{n} / \omega_{n} \sim g_{n} / \psi_{n}$, if $f_{n} * \psi_{m}=g_{m} * \omega_{n}$, for all $n, m \in \mathbb{N}$.

The relation $\sim$ is an equivalent relation on $\mathscr{A}$. The equivalence class containing $f_{n} / \omega_{n}$ is denoted by $\left[f_{n} / \omega_{n}\right]$. These equivalence classes are called Boehmians. The space of all Boehmians is denoted by $\beta_{1}$.

The sum of two Boehmians and multiplication by a scalar can be defined in a natural way

$$
\begin{aligned}
{\left[\frac{f_{n}}{\omega_{n}}\right]+\left[\frac{g_{n}}{\psi_{n}}\right] } & =\left[\frac{f_{n} * \psi_{n}+g_{n} * \omega_{n}}{\omega_{n} * \psi_{n}}\right], \\
\alpha\left[\frac{f_{n}}{\omega_{n}}\right] & =\left[\alpha \frac{f_{n}}{\omega_{n}}\right]=\left[\frac{\alpha f_{n}}{\omega_{n}}\right],
\end{aligned}
$$

$\alpha \in \mathbb{C}$, space of complex numbers.

The operation $*$ and the differentiation are defined by $\left[f_{n} / \omega_{n}\right] *\left[g_{n} / \psi_{n}\right]=\left[\left(f_{n} * g_{n}\right) /\left(\omega_{n} * \psi_{n}\right)\right]$ and $\mathscr{D}^{\alpha}\left[f_{n} / \omega_{n}\right]=$ $\left[\mathscr{D}^{\alpha} f_{n} / \omega_{n}\right]$.

The operation $*$ can be extended to $\beta \times \mathcal{S}$ as follows. If $\left[f_{n} / \omega_{n}\right] \in \beta_{1}$ and $\omega \in \mathcal{S}$, then

$$
\left[\frac{f_{n}}{\omega_{n}}\right] * \omega=\left[\frac{f_{n} * \omega}{\omega_{n}}\right] .
$$


$\delta$-Convergence. A sequence of Boehmians $\left(\beta_{n}\right)$ in $\beta_{1}$ is said to be $\delta$ convergent to a Boehmian $\beta$ in $\beta_{1}$, denoted by $\beta_{n} \stackrel{\delta}{\rightarrow} \beta$, if there exists a delta sequence $\left(\omega_{n}\right)$ such that

$$
\begin{array}{r}
\left(\beta_{n} * \omega_{n}\right),\left(\beta * \omega_{n}\right) \in \mathscr{G}, \quad \forall k, n \in \mathbb{N}, \\
\left(\beta_{n} * \omega_{k}\right) \longrightarrow\left(\beta * \omega_{k}\right) \quad \text { as } n \longrightarrow \infty, \text { in } \mathscr{G}, \\
\text { for every } k \in \mathbb{N} .
\end{array}
$$

The following is equivalent for the statement of $\delta$ convergence: $\beta_{n} \stackrel{\delta}{\rightarrow} \beta(n \rightarrow \infty)$ in $\beta_{1}$ if and only if there is $f_{n, k}, f_{k} \in \mathscr{G}$ and $\left(\omega_{k}\right) \in \Delta$ such that $\beta_{n}=\left[f_{n, k} / \omega_{k}\right]$, $\beta=\left[f_{k} / \omega_{k}\right]$ and for each $k \in \mathbb{N}, f_{n, k} \rightarrow f_{k}$ as $n \rightarrow \infty$ in $\mathscr{G}$.

A sequence of Boehmians $\left(\beta_{n}\right)$ in $\beta_{1}$ is said to be $\Delta$ convergent to a Boehmian $\beta$ in $\beta_{1}$, denoted by $\beta_{n} \stackrel{\Delta}{\rightarrow} \beta$, if there exists a $\left(\omega_{n}\right) \in \Delta$ such that $\left(\beta_{n}-\beta\right) * \omega_{n} \in \beta_{1}$, for all $n \in \mathbb{N}$, and $\left(\beta_{n}-\beta\right) * \omega_{n} \rightarrow 0$ as $n \rightarrow \infty$ in $\beta_{1}$. See [2, 5-15].

\section{Modified Mellin Transform of Boehmian}

In this section we discuss the modified Mellin transform on spaces of Boehmians. Consider the group $\dot{\mu}_{a, b}\left(\mathbb{R}_{+}\right)$and $\vartheta\left(\mathbb{R}_{+}\right)$ as a subgroup of $\mu_{a, b}\left(\mathbb{R}_{+}\right)$. Let $\vee$ be the operation between $\dot{\mu}_{a, b}\left(\mathbb{R}_{+}\right)$and $\vartheta\left(\mathbb{R}_{+}\right)$and $\Delta$ the set of delta sequences given by [2]

(1) $\int_{\mathbb{R}_{+}} \varphi_{n}(t) d t=1$, for all $n \in \mathbb{N}$;

(2) $\int_{\mathbb{R}_{+}}\left|\varphi_{n}(t)\right| d t \leq \mathfrak{m}$, for all $n \in \mathbb{N}$, for some $\mathfrak{m}>0$;

(3) supp $\varphi_{n} \subset\left(a_{n}, b_{n}\right)$, for all $n \in \mathbb{N}$ for some $0<a_{n}<$ $b_{n}<\infty$ with $a_{n} \rightarrow 1, b_{n} \rightarrow 1$ as $n \rightarrow \infty$.

Let $\beta_{1}$ be the Boehmian space obtained from $\mu_{a, b}, \vartheta\left(\mathbb{R}_{+}\right)$and $\Delta$; then $\beta_{1}$ will serve as the domain space of $\mu^{2}$.

Our next objective is to construct a range space, say $\beta_{2}$, for $\mu^{m}$.

Let

$$
\begin{aligned}
\Delta_{\mathbb{R}_{+}}^{e} & =\left\{\mu_{\varphi_{n}}:\left(\varphi_{n}\right) \in \Delta\right\}, \quad \vartheta^{e}=\left\{\mu_{\varphi}: \varphi \in \vartheta\right\}, \\
\ell^{m} & =\left\{\widehat{\mu_{f}^{m}}: f \in \dot{\mu}_{a, b}\right\} .
\end{aligned}
$$

For $g \in \ell^{m}, \psi \in \mathcal{V}^{e}$, define

$$
(g \wedge \psi)(y)=g(y) \psi(y) \text {. }
$$

We have the following theorem.

Theorem 3. Let $g \in \ell^{m}$ and $\psi \in \mathcal{\vartheta}^{e}$, then $g \wedge \psi \in \ell^{m}$.

Proof. Let $g$ and $\psi$ belong to $\ell^{m}$ and $\vartheta^{e}$; respectively. Then there are $f \in \dot{\mu}_{a, b}$ and $\varphi \in \vartheta$ such that $g=\mu_{f}^{m}$ and $\psi=\mu_{\varphi}$, respectively. Therefore, by the convolution theorem and (19) we get that

$$
\begin{aligned}
(g \wedge \psi)(y) & =g(y) \psi(y) \\
& =\left(\widehat{\mu_{f}^{m}} \mu_{\varphi}\right)(y) \\
& =\mu^{m}(f \vee \varphi)(y) .
\end{aligned}
$$

Since $f \vee \varphi \in \dot{\mu}_{a, b}$, it follows $g \wedge \psi \in \ell^{m}$.

Hence we have proved the theorem.

Theorem 4. Let $\psi_{1}, \psi_{2} \in \mathcal{\vartheta}^{e}$; then $\psi_{1} \wedge \psi_{2} \in \vartheta^{e}$.

Proof. By definition of $\vartheta^{e}$ we can find $\varphi_{1}, \varphi_{2} \in \mathcal{\vartheta}$ such that $\psi_{1}=\mu_{\varphi_{1}}$ and $\psi_{2}=\mu_{\varphi_{2}}$.

Therefore, by [1],

$$
\begin{aligned}
\psi_{1} \wedge \psi_{2} & =\psi_{1}(y) \psi_{2}(y) \\
& =\mu_{\varphi_{1}}(y) \mu_{\varphi_{2}}(y) \\
& =\mu\left(\varphi_{1} \vee \varphi_{2}\right)(y) .
\end{aligned}
$$

But since $\varphi_{1} \vee \varphi_{2} \in \vartheta$, we get $\psi_{1} \wedge \psi_{2} \in \vartheta^{e}$. Thus we have the theorem.

Theorem 5. Let $g_{1}, g_{2} \in \ell^{m}$ and $\psi \in \vartheta^{e}$; then $\left(g_{1}+g_{2}\right) \wedge \psi=$ $g_{1} \wedge \psi+g_{2} \wedge \psi$ and $(\alpha g) \wedge \psi=g \wedge(\alpha \psi)=\alpha\left(g_{1} \wedge \psi\right)$.

Proof. Is straightforward.

Theorem 6. Let $g_{n} \rightarrow g$ in $\ell^{m}, \psi \in \vartheta^{e}$; then $g_{n} \wedge \psi \rightarrow g \wedge \psi$ as $n \rightarrow \infty$ in $\ell^{m}$.

Proof. Can easily be checked.

Theorem 7. Let $g \in \ell^{m}$ and $\left(\psi_{n}\right) \in \Delta_{\mathbb{R}_{+}}^{e}$; then $g \wedge \psi_{n} \rightarrow g$ as $n \rightarrow \infty$.

Proof. By (19) we have

$$
\left(g \wedge \psi_{n}\right)(y)=\left(\widehat{\mu_{f}^{m}} \mu_{\varphi_{n}}\right)(y)
$$

where $f \in \dot{\mu}_{a, b}$ and $\left(\varphi_{n}\right) \in \Delta$ are such that $\widehat{\mu_{f}^{m}}=g$ and $\mu_{\varphi_{n}}=$ $\psi_{n}$, for all $n \in \mathbb{N}$.

Since $\mu_{\varphi_{n}}(y) \rightarrow 1$ as $n \rightarrow \infty$ on compact subsets of $\mathbb{R}_{+}$, (22) implies that $\left(g \wedge \psi_{n}\right)(y) \rightarrow \widehat{\mu_{f}^{m}}(y)=g(y)$, for all $y$, as $n \rightarrow \infty$. Hence we obtain the theorem.

Theorem 8. Let $\left(\psi_{n}\right),\left(\theta_{n}\right) \in \Delta_{\mathbb{R}_{+}}^{e}$; then $\psi_{n} \wedge \theta_{n} \in \Delta_{\mathbb{R}_{+}}^{e}$.

Let $\psi_{n}=\mu_{\alpha_{n}}, \theta_{n}=\mu_{\sigma_{n}}$; then taking into account the fact that $\left(\psi_{n} \wedge \theta_{n}\right)(y)=\left(\mu_{\alpha_{n}} \wedge \mu_{\sigma_{n}}\right)(y)=\mu\left(\alpha_{n} \vee \sigma_{n}\right)(y)$, since $\alpha_{n} \vee \sigma_{n} \in \Delta$, this theorem follows.

The Boehmian space $\beta_{2}$ is therefore constructed.

In addition, scalar multiplication, differentiation, and the operation $\wedge$ in $\beta_{2}$ are defined similar to that of usual Boehmian spaces. 
Each $g \in \ell^{m}$ can be identified by a member of $\beta_{2}$ given as

$$
g \longrightarrow\left[\frac{g \wedge \psi_{n}}{\psi_{n}}\right] \quad \text { as } n \longrightarrow \infty,
$$

where $\psi_{n} \in \Delta_{\mathbb{R}_{+}}^{e}$.

Definition 9. The extended modified Mellin transform $\mu^{m}$ : $\beta_{1} \rightarrow \beta_{2}$ is defined by

$$
\widetilde{\mu^{m}}(\beta)=\left[\frac{\widehat{\mu_{f_{n}}^{m}}}{\mu_{\varphi_{n}}}\right], \quad \forall \beta=\left[\frac{f_{n}}{\varphi_{n}}\right] \in \beta_{1} .
$$

Theorem 10. The extended modified Mellin transform is well defined.

Proof. The proof of this theorem is straightforward. See [1113].

Theorem 11 (consistency theorem). The extended modified

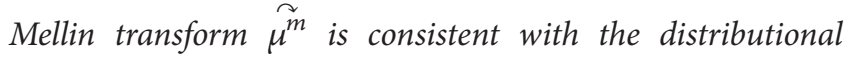
$\widehat{\mu_{f}^{m}}\left(\widehat{\mu_{f}^{m}}: \dot{\mu}_{a, b} \rightarrow \dot{\mu}_{a, b}\right)$.

Proof. For every $f \in \dot{\mu}_{a, b}$, let $\beta$ be its representative in $\beta_{1}$; then $\beta=\left[\left(f \vee \varphi_{n}\right) / \varphi_{n}\right]$, where $\varphi_{n} \in \Delta$, for all $n$. Then it is clear that $\varphi_{n}$ is independent of the representative, for all $n$.

Therefore

$$
\begin{aligned}
\stackrel{2}{\mu^{m}}(\beta) & =\stackrel{\mu^{m}}{\mu^{\prime}}\left(\left[\frac{f \vee \varphi_{n}}{\varphi_{n}}\right]\right) \\
\text { i.e. } & =\left[\frac{\widehat{\mu_{f}^{m}} \mu_{\varphi_{n}}}{\mu_{\varphi_{n}}}\right] \\
\text { i.e. } & =\left[\frac{\left(\widehat{\mu_{f}^{m}}\right) \mu_{\varphi_{n}}}{\mu_{\varphi_{n}}}\right]
\end{aligned}
$$

which is the representative of $\widehat{\mu_{f}^{m}}$ in $\dot{\mu}_{a, b}$.

Hence we have the proof.

Theorem 12 (necessity theorem). Let $\left[g_{n} / \psi_{n}\right] \in \beta_{2}$; then the necessary and sufficient condition that $\left[g_{n} / \psi_{n}\right]$ is to be in the range of $\mu^{2}$ is that $g_{n}$ belongs to range of $\widehat{\mu_{f}^{m}}$ for every $n \in \mathbb{N}$.

Proof. Let $\left[g_{n} / \psi_{n}\right]$ be in the range of $\mu^{\stackrel{m}{m}}$; then of course $g_{n}$ belongs to the range of $\left(\widehat{\mu_{f}^{m}}\right)$, for all $n \in \mathbb{N}$.

To establish the converse, let $g_{n}$ be in the range of $\widehat{\mu_{f}^{m}}$, for all $n \in \mathbb{N}$. Then there is $f_{n} \in \dot{\mu}_{a, b}$ such that $\widehat{\mu_{f_{n}}^{m}}=g_{n}, n \in \mathbb{N}$.

Since $\left[g_{n} / \psi_{n}\right] \in \beta_{2}$,

$$
g_{n} \vee \psi_{m}=g_{m} \vee \psi_{n},
$$

for all $m, n \in \mathbb{N}$. Therefore,

$$
\mu^{m}\left(f_{n} \wedge \varphi_{n}\right)=\mu^{m}\left(f_{m} \wedge \varphi_{n}\right), \quad \forall m, n \in \mathbb{N},
$$

where $f_{n} \in \dot{\mu}_{a, b}$ and $\varphi_{n} \in \Delta$.
The fact that $\widehat{\mu_{f}^{m}}$ is injective, $\widehat{\mu_{f}^{m}}: \dot{\mu}_{a, b} \rightarrow \dot{\mu}_{a, b}$, implies that $f_{n} \wedge \varphi_{m}=f_{m} \wedge \varphi_{n}, m, n \in \mathbb{N}$.

Thus $f_{n} / \varphi_{n}$ is quotient of sequences in $\beta_{1}$. Hence, $\left[f_{n} / \varphi_{n}\right] \in \beta_{1}$ and

$$
\stackrel{\curvearrowright}{\mu^{m}}\left(\left[\frac{f_{n}}{\varphi_{n}}\right]\right)=\left[\frac{g_{n}}{\psi_{n}}\right] .
$$

Hence the theorem is proved.

Theorem 13 (generalized convolution theorem). Let $\beta=$ $\left[f_{n} / \varphi_{n}\right] \in \beta_{1}$ and $\gamma=\left[\kappa_{n} / \phi_{n}\right] \in \beta_{1}$; then

$$
\stackrel{\curvearrowright}{\mu^{m}}(\beta \vee \gamma)=\stackrel{\curvearrowright}{\mu^{m}}\left(\left[\frac{f_{n}}{\varphi_{n}}\right]\right) \wedge \stackrel{2}{\mu^{m}}\left(\left[\frac{\kappa_{n}}{\phi_{n}}\right]\right) .
$$

Proof. Assume that the requirements of the theorem satisfy for some $\beta$ and $\gamma \in \beta_{1}$; then using Definition 9 and the operation $\wedge$ yields

$$
\begin{aligned}
\stackrel{2}{\mu^{m}(\beta \vee \gamma)} & =\stackrel{2}{\mu^{m}}\left(\left[\frac{f_{n} \vee \kappa_{n}}{\varphi_{n} \vee \phi_{n}}\right]\right)=\left[\frac{\mu^{m}\left(f_{n} \vee \kappa_{n}\right)}{\mu\left(\varphi_{n} \vee \phi_{n}\right)}\right] \\
& =\left[\frac{\widehat{\mu_{f_{n}}^{m}} \wedge \mu_{\kappa_{n}}^{m}}{\mu_{\varphi_{n}} \wedge \mu_{\phi_{n}}}\right]=\left[\frac{\widehat{\mu_{f_{n}}^{m}}}{\mu_{\varphi_{n}}}\right] \wedge\left[\frac{\mu_{\kappa_{n}}^{m}}{\mu_{\phi_{n}}}\right] .
\end{aligned}
$$

Therefore

$$
\stackrel{\curvearrowright}{\mu^{m}}(\beta \vee \gamma)=\stackrel{\curvearrowright}{\mu^{m}}\left(\left[\frac{f_{n}}{\varphi_{n}}\right]\right) \wedge \stackrel{\curvearrowright}{\mu^{m}}\left(\left[\frac{\kappa_{n}}{\phi_{n}}\right]\right) .
$$

This completes the proof.

Theorem 14. The extended modified Mellin transform $\mu^{m}$ : $\beta_{1} \rightarrow \beta_{2}$ is bijective.

Proof. Assume $\stackrel{2}{m}^{m}\left[f_{n} / \varphi_{n}\right]=\overbrace{\mu^{m}}^{2}\left[\kappa_{n} / \phi_{n}\right]$; then it follows from the concept of quotients of sequences that $\widehat{\mu_{f_{n}}^{m}} \wedge \mu_{\phi_{m}}=$ $\widehat{\mu_{\kappa_{m}}^{m}} \wedge \mu_{\varphi_{n}}$. Therefore, $\mu^{m}\left(f_{n} \vee \phi_{m}\right)=\mu^{m}\left(\kappa_{m} \vee \varphi_{n}\right)$. The property that $\mu^{m}$ is one to one implies $f_{n} \vee \phi_{m}=\kappa_{m} \vee \varphi_{n}$. Therefore,

$$
\left[\frac{f_{n}}{\varphi_{n}}\right]=\left[\frac{\kappa_{n}}{\phi_{n}}\right] \text {. }
$$

Next to establish that $\stackrel{\curvearrowright}{\mu}^{m}$ is onto, let $\left[\widehat{\mu_{f_{n}}^{m}} / \mu_{\varphi_{n}}\right]\left(\in \beta_{2}\right)$ be arbitrary; then $\widehat{\mu_{f_{n}}^{m}} \wedge \mu_{\varphi_{m}}=\widehat{\mu_{f_{m}}^{m}} \wedge \mu_{\varphi_{n}}$ for every $m, n \in \mathbb{N}$. Hence $f_{n}, f_{m} \in \dot{\mu}_{a, b}$ are such that $\mu^{m}\left(f_{n} \vee \varphi_{m}\right)=\mu^{m}\left(f_{m} \vee \varphi_{n}\right)$, for all $m, n \in \mathbb{N}$.

Hence, the Boehmian $\left[f_{n} / \varphi_{n}\right]$ belongs to $\beta_{1}$ and satisfies

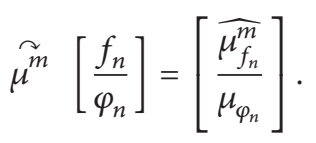

This completes the proof of the theorem. 
Now we introduce $\left(\stackrel{\curvearrowright}{\mu^{m}}\right)^{-1}$ as the inverse transform of $\mu^{m}$, where

$$
\left({\stackrel{\curvearrowright}{\mu^{m}}}^{-1}\left(\left[\frac{\widehat{\mu_{f_{n}}^{m}}}{\mu_{\varphi_{n}}}\right]\right)=\left[\frac{\left(\widehat{\mu_{f_{n}}^{m}}\right)^{-1}\left(\widehat{\mu_{f_{n}}^{m}}\right)}{\left(\mu_{\varphi_{n}}\right)^{-1}\left(\mu_{\varphi_{n}}\right)}\right],\right.
$$

for every $\left[f_{n} / \varphi_{n}\right] \in \beta_{1}$.

Theorem 15. Let $\left[\widehat{\mu_{f_{n}}^{m}} / \mu_{\varphi_{n}}\right] \in \beta_{2}$ and $\phi \in \vartheta^{e}$, then

$$
\begin{gathered}
\left(\stackrel{\curvearrowright}{\mu^{m}}\right)^{-1}\left(\left[\frac{\widehat{\mu_{f_{n}}^{m}}}{\mu_{\varphi_{n}}}\right] \wedge \phi\right)=\left[\frac{f_{n}}{\varphi_{n}}\right] \vee \phi, \\
\stackrel{\curvearrowright}{\mu^{m}}\left(\left[\frac{f_{n}}{\varphi_{n}}\right] \vee \phi\right)=\left[\frac{\widehat{\mu_{f_{n}}^{m}}}{\mu_{\varphi_{n}}}\right] \wedge \phi .
\end{gathered}
$$

Proof. We prove (35) and omit the proof of (36) due to its similarity. Given $\left[\widehat{\mu_{f_{n}}^{m}} / \mu_{\varphi_{n}}\right] \in \beta_{2}$ and $\psi \in \vartheta$ such that $\phi=\mu_{\psi}^{m}$ then employing (34) yields

$$
\begin{aligned}
\left({ }^{m}\right)^{-1}\left(\left[\frac{\widehat{\mu_{f_{n}}^{m}}}{\mu_{\varphi_{n}}}\right] \wedge \phi\right) & =\left({ }_{\mu^{m}}^{\text {m }}\right)^{-1}\left(\left[\frac{\widehat{\mu_{f_{n}}^{m}} \wedge \phi}{\mu_{\varphi_{n}}}\right]\right) \\
& =\left[\frac{\left(\mu_{f_{n}}^{m}\right)^{-1}\left(\widehat{\mu_{f_{n}}^{m}} \wedge \mu_{\psi}^{m}\right)}{\left(\mu_{\varphi_{n}}\right)^{-1}\left(\mu_{\varphi_{n}}\right)}\right] .
\end{aligned}
$$

Using (19) gives

$$
\left(\mu^{m}\right)^{-1}\left(\left[\frac{\widehat{\mu_{f_{n}}^{m}}}{\mu_{\varphi_{n}}}\right] \wedge \phi\right)=\left[\frac{\left(\mu_{f_{n}}^{m}\right)^{-1}\left(\widehat{\mu_{f_{n}}^{m}} \mu_{\psi}^{m}\right)}{\left(\mu_{\varphi_{n}}\right)^{-1}\left(\mu_{\varphi_{n}}\right)}\right] .
$$

Hence the convolution theorem gives

$$
\left(\widehat{\curvearrowright}^{m}\right)^{-1}\left(\left[\frac{\widehat{\mu_{f_{n}}^{m}}}{\mu_{\varphi_{n}}}\right] \wedge \phi\right)=\left[\frac{\left(\mu_{f_{n}}^{m}\right)^{-1}\left(\mu_{f_{n}}^{m}\left(f_{n} \vee \phi\right)\right)}{\left(\mu_{\varphi_{n}}\right)^{-1}\left(\mu_{\varphi_{n}}\right)}\right] .
$$

Thus

$$
\left(\stackrel{\curvearrowright}{\mu^{m}}\right)^{-1}\left(\left[\frac{\widehat{\mu_{f_{n}}^{m}}}{\mu_{\varphi_{n}}}\right] \wedge \phi\right)=\left[\frac{f_{n}}{\varphi_{n}}\right] \vee \phi .
$$

Proof of the second part is similar.

This completes the proof of the theorem.

Theorem 16. $\stackrel{\curvearrowright}{\mu^{m}}: \beta_{1} \rightarrow \beta_{2}$ and $\left(\stackrel{\curvearrowright}{\mu^{m}}\right)^{-1}: \beta_{2} \rightarrow \beta_{1}$ are continuous with respect to $\delta$-convergence.

Proof. Let $\beta_{n} \stackrel{\delta}{\rightarrow} \beta$ in $\beta_{1}$ as $n \rightarrow \infty$; then we establish that $\mu^{m} \beta_{n} \rightarrow \mu^{m} \beta$ as $n \rightarrow \infty$. Let $f_{n, k}$ and $f_{k}$ be in $\mu_{a, b}$ such that

$$
\beta_{n}=\left[\frac{f_{n, k}}{\varphi_{k}}\right], \quad \beta=\left[\frac{f_{k}}{\varphi_{k}}\right]
$$

and $f_{n, k} \rightarrow f_{k}$ as $n \rightarrow \infty$ for every $k \in \mathbb{N}$. Thus,

The continuity of $\widehat{\mu_{f_{n, k}^{m}}^{m}}$ implies $\widehat{\mu_{f_{n, k}}^{m}} \rightarrow \widehat{\mu_{f_{k}}^{m}}$ as $n \rightarrow \infty$.

$$
\left[\begin{array}{c}
\widehat{\mu_{f_{n, k}}^{m}} \\
\mu_{\varphi_{k}}
\end{array}\right] \rightarrow\left[\begin{array}{c}
\widehat{\mu_{f_{k}}^{m}} \\
\mu_{\varphi_{k}}
\end{array}\right]
$$

as $n \rightarrow \infty$ in $\beta_{2}$. This proves continuity of $\stackrel{\curvearrowright}{m}^{m}$.

Next, let $g_{n} \stackrel{\delta}{\rightarrow} g \in \beta_{2}$ as $n \rightarrow \infty$; then we have $g_{n}=$ $\left[\widehat{\mu_{f_{n, k}}^{m}} / \mu_{\varphi_{k}}\right]$ and $g=\left[\widehat{\mu_{f_{k}}^{m}} / \mu_{\varphi_{k}}\right]$ for some $\widehat{\mu_{f_{n, k}}^{m}}, \widehat{\mu_{f_{k}}^{m}} \in \ell^{m}$, where $\widehat{\mu_{f_{n, k}}^{m}} \rightarrow \widehat{\mu_{f_{k}}^{m}}$ as $n \rightarrow \infty$. Hence

$$
\left(\widehat{\mu_{f_{n, \mathrm{k}}}^{m}}\right)^{-1}\left(\widehat{\mu_{f_{n, k}}^{m}}\right) \longrightarrow\left(\widehat{\mu_{f_{k}}^{m}}\right)^{-1}\left(\widehat{\mu_{f_{k}}^{m}}\right)
$$

as $n \rightarrow \infty$ in $\beta_{1}$. That is,

$$
\begin{aligned}
{\left[\frac{\left(\widehat{\mu_{f_{n, k}}^{m}}\right)^{-1}\left(\widehat{\mu_{f_{n, k}}^{m}}\right)}{\varphi_{k}}\right] } & =\left[\frac{\left(\widehat{\mu_{f_{n, k}}^{m}}\right)^{-1}\left(\widehat{\mu_{f_{n, k}}^{m}}\right)}{\left(\mu_{\varphi_{k}}\right)^{-1}\left(\mu_{\varphi_{k}}\right)}\right] \\
& \longrightarrow\left[\frac{\left(\widehat{\mu_{f_{k}}^{m}}\right)^{-1}\left(\widehat{\mu_{f_{k}}^{m}}\right)}{\varphi_{k}}\right]
\end{aligned}
$$

as $n \rightarrow \infty$.

Hence

$$
\left[\frac{\left(\widehat{\mu_{f_{n, k}}^{m}}\right)^{-1}\left(\widehat{\mu_{f_{n, k}}^{m}}\right)}{\varphi_{k}}\right] \rightarrow\left[\frac{\left(\widehat{\mu_{f_{k}}^{m}}\right)^{-1}\left(\widehat{\mu_{f_{k}}^{m}}\right)}{\left(\mu_{\varphi_{k}}\right)^{-1}\left(\mu_{\varphi_{k}}\right)}\right]
$$

as $n \rightarrow \infty$.

That is,

$$
\left(\stackrel{\curvearrowright}{\mu^{m}}\right)^{-1} g_{n} \longrightarrow\left({\stackrel{\curvearrowright}{\mu^{m}}}^{-1} g\right.
$$

as $n \rightarrow \infty$. This completes the proof.

Theorem 17. $\stackrel{\curvearrowright}{m}^{m}: \beta_{1} \rightarrow \beta_{2}$ and $\left(\stackrel{\curvearrowright}{m}^{-1}: \beta_{2} \rightarrow \beta_{1}\right.$ are continuous with respect to $\Delta$-convergence.

Proof. Let $\beta_{n} \stackrel{\Delta}{\rightarrow} \beta$ in $\beta_{1}$ as $n \rightarrow \infty$. Then, we find $f_{n} \in \dot{\mu}_{a, b}$ and $\left(\varphi_{k}\right) \in \Delta$ such that $\left(\beta_{n}-\beta\right) \wedge \varphi_{k}=\left[\left(f_{n} \wedge \varphi_{k}\right) / \varphi_{k}\right]$ and $f_{n} \rightarrow 0$ as $n \rightarrow \infty$. Therefore

$$
\stackrel{\curvearrowright}{\mu^{m}}\left(\left(\beta_{n}-\beta\right) \vee \varphi_{k}\right)=\left[\frac{\mu^{m}\left(f_{n} \vee \varphi_{k}\right)}{\mu_{\varphi_{k}}}\right] .
$$

Hence, $\stackrel{\curvearrowright}{m}^{m}\left(\left(\beta_{n}-\beta\right) \vee \varphi_{k}\right)=\left[\left(\widehat{\mu_{f_{n}}^{m}} \wedge \mu_{\varphi_{k}}\right) / \mu_{\varphi_{k}}\right]=\widehat{\mu_{f_{n}}^{m}} \rightarrow 0$ as $n \rightarrow \infty$ in $\ell^{m}$.

Therefore

$$
\begin{aligned}
& \stackrel{\curvearrowright}{\mu^{m}}\left(\left(\beta_{n}-\beta\right) \vee \varphi_{n}\right) \\
& \quad=\left(\mu^{2} \beta_{n}-\mu^{m} \beta\right) \wedge \mu_{\varphi_{k}} \longrightarrow \quad \text { as } n \longrightarrow \infty .
\end{aligned}
$$

Hence, $\stackrel{\curvearrowright}{\mu^{m}} \beta_{n} \stackrel{\Delta}{\rightarrow} \stackrel{\curvearrowright}{\mu^{m}} \beta$ as $n \rightarrow \infty$. 
Proof of the second part is analogous. Detailed proof is as follows.

Finally, let $g_{n} \stackrel{\Delta}{\rightarrow} g$ in $\beta_{2}$ as $n \rightarrow \infty$; then we can find $\widehat{\mu_{f_{k}}^{m}} \in \ell^{m}$ such that $\left(g_{n}-g\right) \wedge \mu_{\varphi_{k}}=\left[\left(\widehat{\mu_{f_{k}}^{m}} \wedge \mu_{\varphi_{k}}\right) / \mu_{\varphi_{k}}\right]$ and $\widehat{\mu_{f_{k}}^{m}} \rightarrow 0$ as $n \rightarrow \infty$ for some $\left(\mu_{\varphi_{k}}\right) \in \Delta_{\mathbb{R}_{+}}^{e}$.

Next, we have

$$
\left(\stackrel{\curvearrowright}{m}^{-1}\left(\left(g_{n}-g\right) \wedge \mu_{\varphi_{k}}\right)=\left[\frac{\left(\mu_{f_{k}}^{m}\right)^{-1}\left(\widehat{\mu_{f_{k}}^{m}} \wedge \mu_{\varphi_{k}}\right)}{\left(\mu_{\varphi_{k}}\right)^{-1}\left(\mu_{\varphi_{k}}\right)}\right] .\right.
$$

Thus, by (34) we get

$$
\left({\stackrel{\curvearrowright}{\mu^{m}}}^{-1}\left(\left(g_{n}-g\right) \wedge \mu_{\varphi_{k}}\right)=\left[\frac{f_{n} \vee \varphi_{k}}{\varphi_{k}}\right]=f_{n} \longrightarrow 0\right.
$$

as $n \rightarrow \infty$ in $\dot{\mu}_{a, b}$.

Therefore

$$
\begin{aligned}
& \left(\stackrel{\curvearrowright}{\mu^{m}}\right)^{-1}\left(\left(g_{n}-g\right) \wedge \mu_{\varphi_{k}}\right) \\
& =\left(\left(\stackrel{\curvearrowright}{\mu^{m}}\right)^{-1} g_{n}-\left(\stackrel{\curvearrowright}{\mu^{m}}\right)^{-1} g\right) \vee \varphi_{k} \longrightarrow 0
\end{aligned}
$$

as $n \rightarrow \infty$.

Thus, we have

$$
\left(\stackrel{\curvearrowright}{\mu^{m}}\right)^{-1} g_{n} \stackrel{\Delta}{\rightarrow}\left(\stackrel{\curvearrowright}{\mu^{m}}\right)^{-1} g
$$

as $n \rightarrow \infty$ in $\beta_{1}$.

This completes the proof of the theorem.

\section{Conflict of Interests}

The authors declare that there is no conflict of interests regarding the publication of this paper.

\section{Acknowledgment}

The authors acknowledge that this research was partially supported by Universiti Putra Malaysia under the ERGS 12013/5527179.

\section{References}

[1] A. H. Zemanian, Generalized Integral Transformations, Dover, New York, NY, USA, 1987.

[2] S. K. Q. Al-Omari, "On the distributional Mellin transformation and its extension to Boehmian spaces," International Journal of Contemporary Mathematical Sciences, vol. 6, no. 17-20, pp. 801810, 2011.

[3] J. Yang, T. K. Sarkar, and P. Antonik, "Applying the Fouriermodified Mellin transform to Doppler-distorted waveforms," Digital Signal Processing, vol. 17, no. 6, pp. 1030-1039, 2007.

[4] S. K. Q. Al-Omari, "A Mellin transform for a space of Lebesgue integrable Boehmians," International Journal of Contemporary Mathematical Sciences, vol. 6, no. 29-32, pp. 1597-1606, 2011.
[5] S. K. Q. Al-Omari, "Distributional and tempered distributional diffraction Fresnel transforms and their extension to Boehmian spaces," Italian Journal of Pure and Applied Mathematics, no. 30, pp. 179-194, 2013.

[6] P. K. Banerji, S. K. Al-Omari, and L. Debnath, "Tempered distributional Fourier sine (cosine) transform," Integral Transforms and Special Functions, vol. 17, no. 11, pp. 759-768, 2006.

[7] P. Mikusiński, "Fourier transform for integrable Boehmians," The Rocky Mountain Journal of Mathematics, vol. 17, no. 3, pp. 577-582, 1987.

[8] P. Mikusiński, “Tempered Boehmians and ultradistributions," Proceedings of the American Mathematical Society, vol. 123, no. 3, pp. 813-817, 1995.

[9] P. Mikusiński, “Convergence of Boehmians," Japanese Journal of Mathematics, vol. 9, no. 1, pp. 159-179, 1983.

[10] S. K. Q. Al-Omari, D. Loonker, P. K. Banerji, and S. L. Kalla, "Fourier sine (cosine) transform for ultradistributions and their extensions to tempered and ultraBoehmian spaces," Integral Transforms and Special Functions, vol. 19, no. 5-6, pp. 453-462, 2008.

[11] S. K. Q. Al-Omari, "Hartley transforms on a certain space of generalized functions," Georgian Mathematical Journal, vol. 20, no. 3, pp. 415-426, 2013.

[12] S. K. Q. Al-Omari and A. Kılıçman, "Note on Boehmians for class of optical Fresnel wavelet transforms," Journal of Function Spaces and Applications, vol. 2012, Article ID 405368, 14 pages, 2012.

[13] S. K. Q. Al-Omari and A. K1lıçman, "On generalized hartleyHilbert and Fourier-Hilbert transforms," Advances in Difference Equations, vol. 2012, article 232, 12 pages, 2012.

[14] T. K. Boehme, “The support of Mikusiński operators," Transactions of the American Mathematical Society, vol. 176, pp. 319-334, 1973.

[15] S. K. Q. Al-Omari and A. Kılıçman, "On diffraction Fresnel transforms for Boehmians," Abstract and Applied Analysis, vol. 2011, Article ID 712746, 11 pages, 2011. 


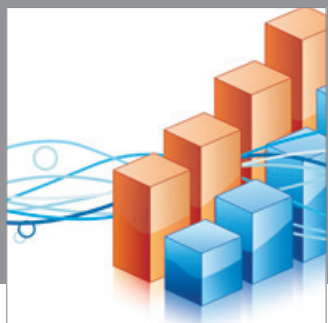

Advances in

Operations Research

mansans

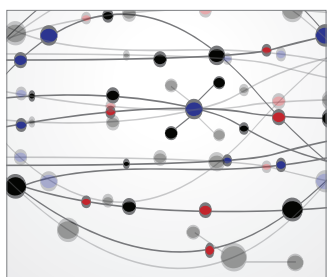

The Scientific World Journal
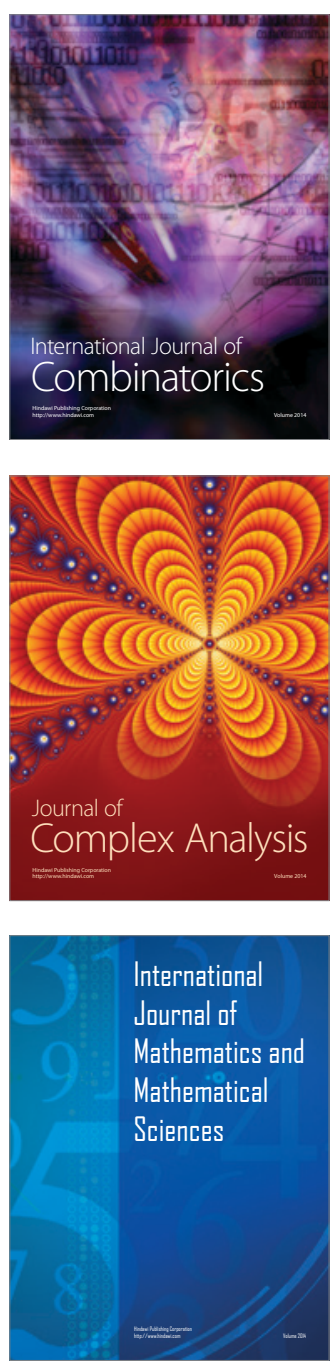
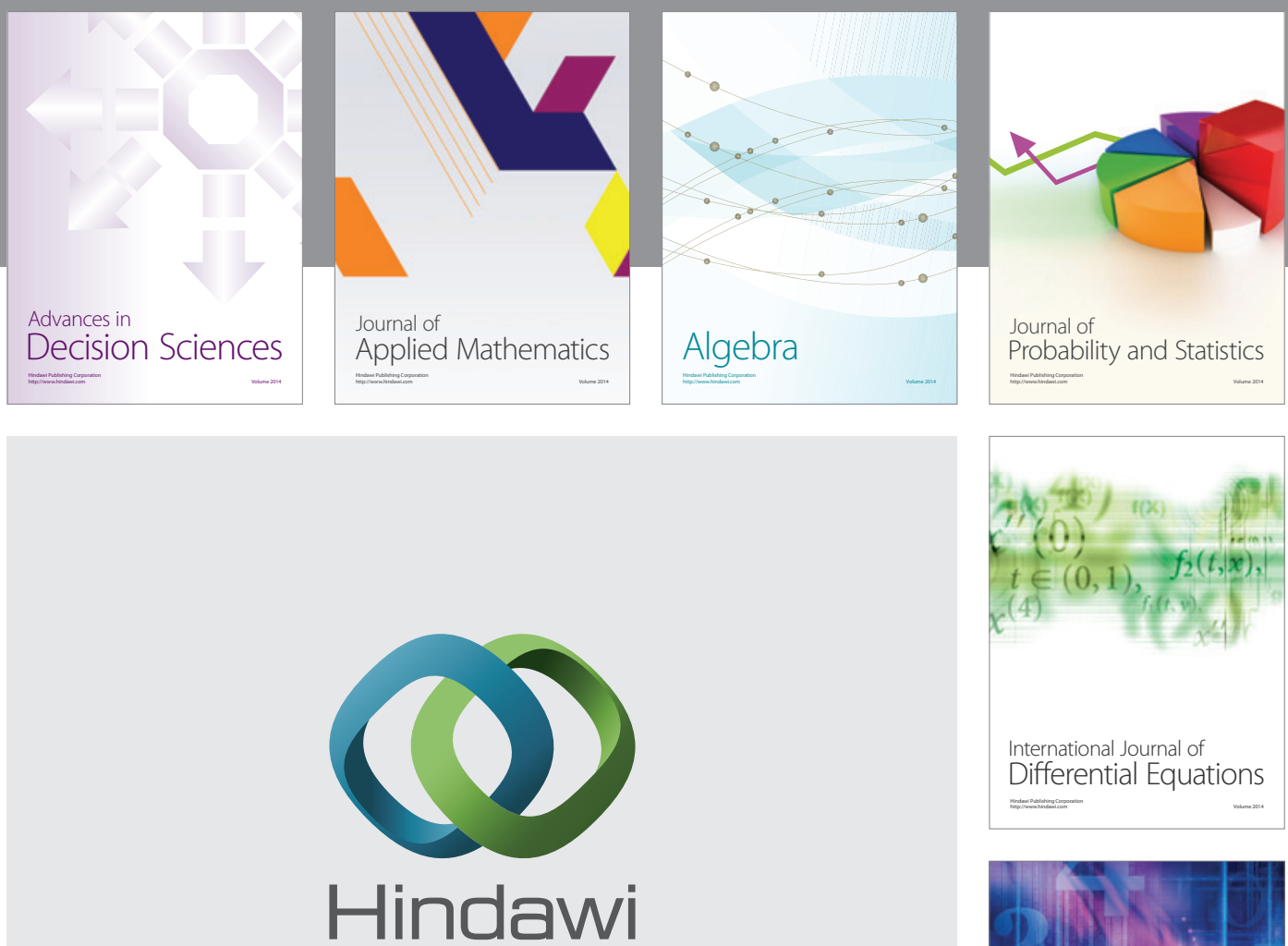

Submit your manuscripts at http://www.hindawi.com
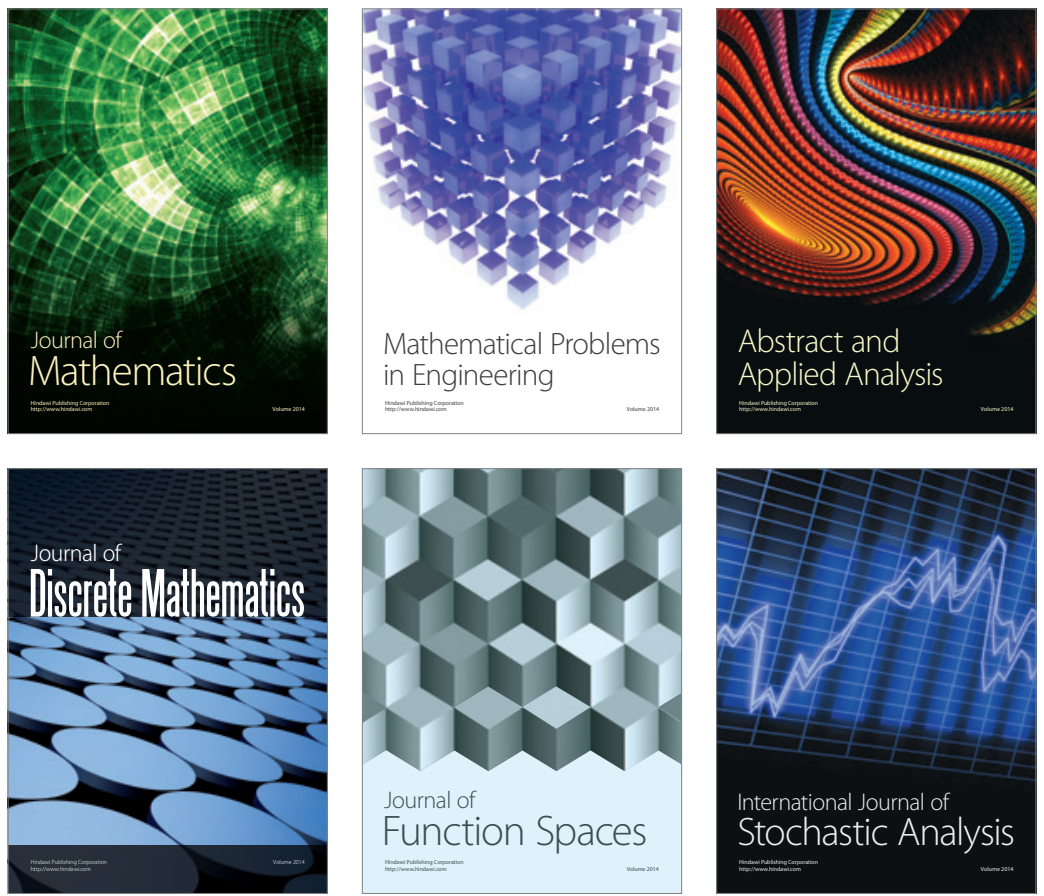

Journal of

Function Spaces

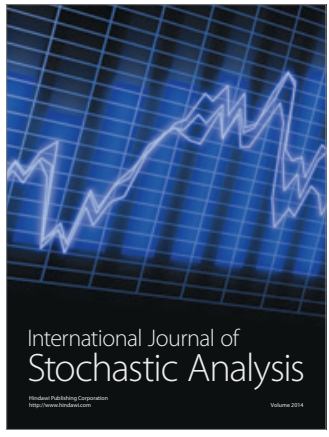

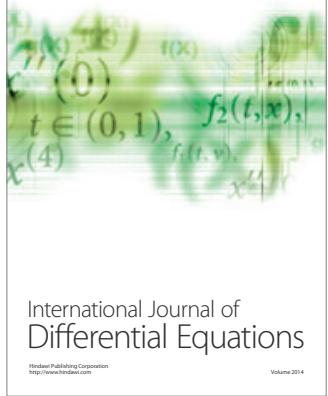
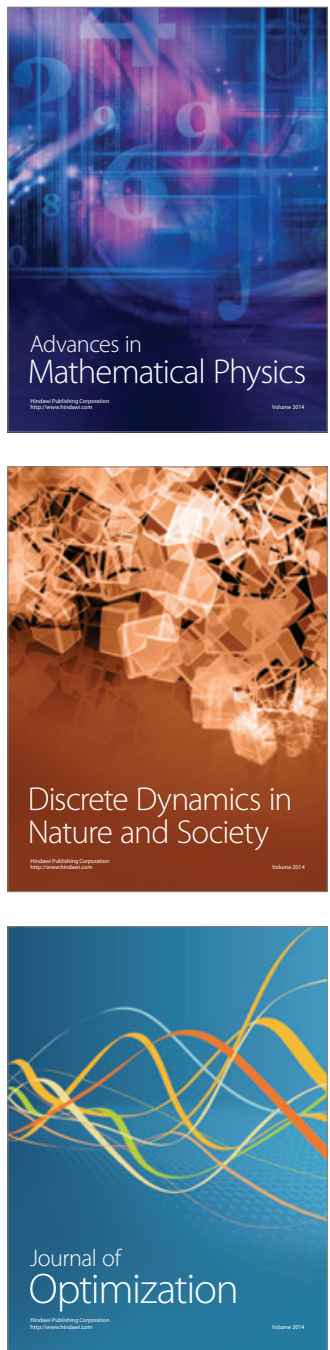